\title{
Probabilistic Reachability Analysis for Large Scale Stochastic Hybrid Systems
}

\author{
Henk A.P. Blom, G.J. (Bert) Bakker and Jaroslav Krystul
}

\begin{abstract}
This paper studies probabilistic reachability analysis for large scale stochastic hybrid systems (SHS) as a problem of rare event estimation. In literature, advanced rare event estimation theory has recently been embedded within a stochastic analysis framework, and this has led to significant novel results in rare event estimation for a diffusion process using sequential MC simulation. This paper presents this rare event estimation theory directly in terms of probabilistic reachability analysis of an SHS, and develops novel theory which allows to extend the novel results for application to a large scale SHS where a very huge number of rare discrete modes may contribute significantly to the reach probability. Essentially, the approach taken is to introduce an aggregation of the discrete modes, and to develop importance sampling relative to the rare switching between the aggregation modes. The practical working of this approach is demonstrated for the safety verification of an advanced air traffic control example.
\end{abstract}

Index Terms - Air transportation, Collision processes, Monte Carlo methods, Risk analysis, Safety, Sequential estimation, Stochastic systems

\section{INTRODUCTION}

$\mathrm{T}$ HIS study is motivated by the problem of safety verification of a future air traffic concept of operation through the analysis of reach probabilities. From a control theoretic perspective such an advanced concept of operations is a blueprint of a controlled Stochastic Hybrid System (SHS). Recently, Sastry and co-workers [1]-[2] studied the optimization of the control policy of a discretetime SHS, such that the probability of staying within some prescribed safe set remains above some prescribed minimum level. [1] developed a theoretical framework which expressed the reach probability as a multiplicative function, and this was used to develop a dynamic programming based approach to compute probabilistic maximal safe sets, i.e. initial states of a system for which control policies exists that assure the reach probability to stay below some given value. [2] showed this problem to be complementary to the problem of how to optimize the control policy of an SHS such that the reach probability of

Henk Blom and Bert Bakker are with National Aerospace Laboratory NLR, PO Box 90502, 1006 BM, Amsterdam, blom@nlr.nl, bakker@nlr.nl. Jaroslav Krystul is with University of Twente, Department of Applied Mathematics, Enschede, The Netherlands, krystulj@ewi.utwente.nl some prescribed unsafe set remains below some given maximum level, and that the same dynamic programming based computation of maximal safe sets can be used. The dynamic programming approach becomes computationally intractable when the SHS considered is of large scale type. Prandini and $\mathrm{Hu}$ [3] developed a Markov chain approximation based method for the computation of reach probabilities for a continuous time SHS. This way the dynamic programming challenge was avoided, however the computational load of their method prohibits application to a large scale SHS.

In theory, reach probability estimation can be done by simulating many trajectories of the process considered, and to count the fraction of cases where the simulated trajectory reaches the unsafe set within some given period $T$. When the reach probability value is very small then the number of straightforward MC simulations needed is impractically large. Rare event estimation literature forms a potentially rich source of information for speeding up MC simulation through combining methods from large deviation and importance sampling theories, e.g. [4], [29]. An early successful development in this area is sequential MC simulation for the estimation of the intensity of radiation that penetrates a shield of absorbing material in nuclear physics, e.g. [5]. More recently this approach has also found application in non-nominal delay time and loss estimation in telecommunication networks, e.g. [6]. L'Ecuyer at al. [7] provide a very good recent overview of these sequential MC simulation developments.

In order to exploit rare event estimation theory within probabilistic reachability analysis of controlled SHS, we are in need of establishing a theoretically unambiguous connection between the two concepts. Implicitly, this connection has recently been elaborated by Del Moral and co-workers [8]-[11]. They embedded theoretical physics equations, which supported the development of advanced MC simulations, within the stochastic analysis setting that is typically used for probabilistic reachability analysis. They subsequently showed that this embedding provides a powerful background for the development and analysis of sequential MC simulation for rare event simulation.

The aim of this paper is to present a part of the framework developed by Del Moral et al. [8]-[11] in a 
probabilistic reachability setting, to further develop this for a large scale SHS, and to demonstrate its practical use for safety verification of an advanced air traffic operation. In [12]-[13], the practical use of the approach of Del Moral [8]-[11] for safety verification of an advanced air traffic operation has already been demonstrated for some specific scenarios. In these scenarios, the main contributions to the reach probability value came from diffusion behavior. It also became clear that the same sequential MC simulation approach failed to work for scenarios of the same air traffic operation where the reach probability is determined by rare switching between modes. This paper is aimed to handle such more demanding rare event estimation problems for large scale controlled SHS. Essentially the approach is to introduce an aggregation of the discrete mode process, and to develop importance sampling approaches for the large scale SHS which work relative to the switching between the aggregated modes.

The paper is organized as follows. Section II develops a factorization of the reach probability. Section III explains the approach of [8]-[11]. Section IV develops the aggregation mode process and characterizes key relations with the controlled SHS. Section V develops a novel sequential MC simulation approach for estimating reach probabilities. Section VI applies this approach towards estimating reach probabilities for an air traffic scenario for which the approach of [8]-[11] falls short.

\section{FACTORIZATION OF REACH PROBABILITY}

Throughout this and the following sections, all stochastic processes are defined on a complete stochastic basis $(\Omega, F$, $\mathbb{F}, \mathrm{P}, \mathrm{T})$ with $(\Omega, F, \mathrm{P})$ a complete probability space, and $\mathbb{F}$ an increasing sequence of sub- $\sigma$-algebra's on the positive time line $\mathrm{T}=\mathbb{R}_{+}$, i.e. $\mathbb{F} \triangleq\left\{\mathcal{J},\left(F_{t}, t \in \mathrm{T}\right), \mathcal{F}\right\}, \mathcal{J}$ containing all P-null sets of $F$ and $\mathcal{J} \subset F_{s} \subset F_{t} \subset \mathcal{F}$ for every $s<t$.

Let us denote $E^{\prime}=\mathbb{R}^{n} \times \mathbb{M}$, with $\mathbb{M}$ a discrete set. Let $\mathcal{E}^{\prime}$ be the Borel $\sigma$-algebra of $E^{\prime}$. We consider a time-homogeneous strong Markov process which also is a generalised stochastic hybrid process $\left\{x_{t}, \theta_{t}\right\}$ [16], [19][21], with $\left\{x_{t}\right\}$ assuming values in $\mathbb{R}^{n}$ and $\left\{\theta_{t}\right\}$ assuming values in $\mathbb{M}$. The first component of $\left\{x_{t}\right\}$ equals $t$ and the other components of $\left\{x_{t}\right\}$ form an $\mathbb{R}^{n-1}$ valued cadlag process $\left\{s_{t}\right\}$. The problem considered is to estimate the probability that $\left\{s_{t}\right\}$ hits a given "small" closed subset $D \subset \mathbb{R}^{n-1}$ within a given time period $[0, T)$, i.e. $P\left(\exists t \in[0, T) ; s_{t} \in D\right)$.
Following Del Moral and co-workers [8]-[11], this probability can be characterized in the form of a multiplicative function the terms of which are defined through an arbitrarily assumed nested sequence of closed subsets $D=D_{m} \subset D_{m-1} \subset \ldots \subset D_{1}$, with the constraint that $P\left(s_{0} \in D_{1}\right)=0$. In order to derive a multiplicative functional characterization of the hitting probability, we set $\tau_{0}=0$ and define $\tau_{k}, k=1, . ., m$, as the first moment that $\left\{s_{t}\right\}$ hits subset $k$, i.e.

$\tau_{k}=\inf \left\{t>0 ; \mathrm{s}_{t} \in D_{k}\right\}$

which implies $P\left(\exists t \in[0, T) ; s_{t} \in D_{m}\right)=P\left(\tau_{m}<T\right)$.

We also define $\{0,1\}$-valued random variables $\left\{\chi_{k}, k=0, . ., m\right\}$ as follows:

$$
\begin{aligned}
\chi_{k} & =1, \quad \text { if } \tau_{k}<T \text { or } k=0 \\
& =0, \text { else. }
\end{aligned}
$$

By using these $\tau_{k}$ and $\chi_{k}$ definitions and the fact that each component of $\left\{s_{t}\right\}$ that may hit any $D_{k}, k=1, . ., m$, has continuous paths, we can write the probability of $\left\{s_{t}\right\}$ hitting $D$ before $T$ as a product of conditional probabilities of reaching $D_{k}$ given $D_{k-1}$ has been reached at some earlier moment in time, i.e.

$$
\begin{aligned}
P\left(\tau_{m}<T\right) & =\mathbb{E}\left[\chi_{m}\right]=\mathbb{E}\left[\prod_{k=1}^{m} \chi_{k}\right]=\prod_{k=1}^{m} \mathbb{E}\left[\chi_{k} \mid \chi_{k-1}=1\right] \\
& =\prod_{k=1}^{m} P\left(\tau_{k}<T \mid \tau_{k-1}<T\right)=\prod_{k=1}^{m} \gamma_{k}
\end{aligned}
$$

with $\gamma_{k} \triangleq P\left(\tau_{k}<T \mid \tau_{k-1}<T\right)$

With this, the problem can be seen as one to estimate the conditional probabilities $\gamma_{k}$ in such a way that the product of the estimators $\tilde{\gamma}_{k}$ is unbiased. Because of the multiplication of the various individual $\tilde{\gamma}_{k}$ estimators, which depend on each other, in general such a product may be heavily biased. Garvels et al. [17]-[18] was the first to show that for a discrete-time Markov process this approach guarantees unbiased estimation. The key novelty of [8]-[11] was to develop a sequential MC simulation approach for the estimation of the $\gamma_{k}$ 's in (2), which guarantees unbiased estimation of $P\left(\tau_{m}<T\right)$ under the condition that $\left\{s_{t}\right\}$ is (or is embedded in) a strong Markov process.

\section{SEQUENTIAL MC SIMULATION}

For the process $\left\{x_{t}, \theta_{t}\right\}$ we follow the approach of [8]- 
[11] to characterize how the evolution proceeds from $\tau_{k-1} \wedge T$ to $\tau_{k} \wedge T$. For any $B \in \mathcal{E}^{\prime}$, let $p_{\xi_{k} \mid \chi_{k^{\prime}}}(B \mid 1)$ denote the conditional probability of $\xi_{k}=\left(x_{\tau_{k} \wedge T}, \theta_{\tau_{k} \wedge T}\right) \in B$ given $\chi_{k^{\prime}}=1$. Under the assumption that $P\left(s_{0} \in D_{1}\right)=0$, we characterize the following recursive sequence of transformations

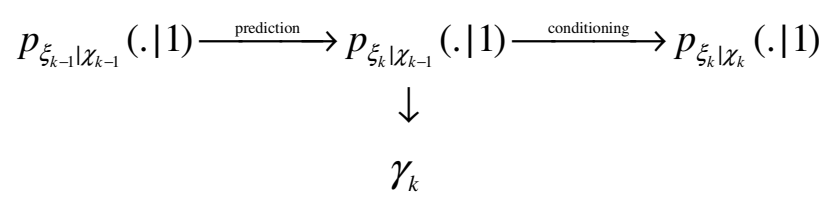

Because $\left\{x_{t}, \theta_{t}\right\}$ is a strong Markov process, $\left\{\xi_{k}\right\}$ is a Markov sequence. Hence the prediction step satisfies a Chapman-Kolmogorov equation:

$p_{\xi_{k} \mid \chi_{k-1}}(B \mid 1)=\int_{E^{\prime}} p_{\xi_{k} \mid \xi_{k-1}}(B \mid \xi) p_{\xi_{k-1} \mid \chi_{k-1}}(d \xi \mid 1)$

Next we characterize the conditional probability of reaching the next subset:

$$
\begin{aligned}
\gamma_{k} & =P\left(\tau_{k}<T \mid \tau_{k-1}<T\right)=P\left(\chi_{k}=1 \mid \chi_{k-1}=1\right)= \\
& =\mathbb{E}\left[\chi_{k} \mid \chi_{k-1}=1\right]=\int_{E^{\prime}} 1_{Q_{k}}(\xi) p_{\xi_{k} \mid \chi_{k-1}}(d \xi \mid 1),
\end{aligned}
$$

where $Q_{k} \triangleq(0, T) \times D_{k} \times \mathbb{M}$.

Similarly, the condition step satisfies, for any $B \in \mathcal{E}^{\prime}$ :

$$
p_{\xi_{k} \mid \chi_{k}}(B \mid 1)=\frac{\int_{B} 1_{Q_{k}}(\xi) p_{\xi_{k} \mid \chi_{k-1}}(d \xi \mid 1)}{\int_{E^{\prime}} 1_{Q_{k}}\left(\xi^{\prime}\right) p_{\xi_{k} \mid \chi_{k-1}}\left(d \xi^{\prime} \mid 1\right)} .
$$

With this, the $\gamma_{k}$ 's in (2) are characterized as a solution of the set of recursive equations (3)-(5). Following [8]-[11], this recursive characterization can numerically be approximated through a sequential MC simulation to estimate $P\left(\tau_{m}<T\right)$. This is referred to as the IPS (Interacting Particle System) algorithm, and works as follows.

Simulate $N_{p}$ random trajectories of $\left\{x_{t}, \theta_{t}\right\}$ over $[0, T)$, each of which starts from a random initial condition $\left(\left(0, s_{0}\right), \theta_{0}\right)$, with $s_{0} \notin D_{1}$. Each simulated trajectory stops at $\tau_{1} \wedge T$, i.e. upon hitting $Q_{1}$ or when the first $x$-component reaches $T$. The full hybrid states of these trajectory end points form an empirical density $\tilde{\pi}_{1}$ as an approximation of $p_{\xi_{1} \mid \chi_{1}}(. \mid 1)$. This empirical density is used to generate (i.e. to resample) $N_{p}$ initial conditions of trajectories which are subsequently simulated until hitting
$Q_{2}$ or when the first $x$-component reaches $T$; the end points in $Q_{2}$ form an empirical density $\tilde{\pi}_{2}$ as an approximation of $p_{\xi_{2} \mid \chi_{2}}(. \mid 1)$. This cycle repeats from $Q_{2}$ to $Q_{3}, \ldots$, and finally from $Q_{m-1}$ to $Q_{m}=Q$. During the $k$-th cycle, a fraction $\tilde{\gamma}_{k}$ of the $N_{p}$ simulated trajectories arrives at $Q_{k}$. The product of these $m$ fractions forms an estimator for $P\left(\tau_{m}<T\right)$.

Using the recursive characterization of the conditional density, [8],[10] have also shown that the product of these fractions $\tilde{\gamma}_{k}$ forms an unbiased estimate of the probability of $\left\{s_{t}\right\}$ to hit the set $D$ within the time period $[0, T)$, i.e.

$$
\mathbb{E}\left[\prod_{k=1}^{m} \tilde{\gamma}_{k}\right]=\prod_{k=1}^{m} \gamma_{k}=P(\tau<T)
$$

In addition there is a bound on the $L^{1}$ estimation error, i.e.:

$$
\mathbb{E}\left(\prod_{k=1}^{m} \tilde{\gamma}_{k}-\prod_{k=1}^{m} \gamma_{k}\right) \leq \frac{c_{p}}{\sqrt{N_{p}}}
$$

with $c_{p}$ a finite constant which depends on the simulated scenario and the sequence of nested subsets adopted. [30] develops some complementary error bounds.

Application of this IPS algorithm to air traffic operation may work well for specific scenarios where rare discrete modes are not significantly contributing to the reach probability [12]-[13]. However, there also are relevant scenarios which do not satisfy the latter condition. In the next section we develop theory to handle such cases.

\section{Aggregation Of Modes}

In [14]-[16], hybrid versions of the baseline IPS algorithm [8]-[11] have been developed, which take into account that rare discrete modes may contribute significantly to the reach probability to be estimated. In [14], the hybrid IPS version simulates another, more frequent switching, $\mathbb{M}$-valued process $\left\{\breve{\theta}_{t}\right\}$ and compensates importance weights for the difference between $\left\{\breve{\theta}_{t}\right\}$ and $\left\{\theta_{t}\right\}$. In [15], the hybrid IPS version uses exact probabilistic equations for the evolution of $\left\{\theta_{t}\right\}$ rather than performing simulations. Both hybrid IPS versions resample at the end of each IPS cycle $N_{p} x$-values from $\tilde{\pi}_{k}(., \theta)$ for each mode $\theta \in \mathbb{M}$, leading to a total of $N_{p} \times \mid \mathbb{M}$ I particles, where $|\mathbb{M}|$ is the number of elements in $\mathbb{M}$. When I MI I is very large, then these hybrid IPS approaches are computationally intractable. 
The idea is to improve the situation for very large | $\mathbb{M} \mid$ through application of the hybrid IPS approaches not to $\left\{\theta_{t}\right\}$ but to a $\mathbb{K}$-valued process $\left\{\kappa_{t}\right\}$ with $|\mathbb{K}| \ll|\mathbb{M}|$. In order to accomplish this, we introduce a partition $\left\{\mathbb{M}_{\kappa}, \kappa \in \mathbb{K}\right\}$, such that $\bigcup_{\kappa \in \mathbb{K}} \mathbb{M}_{\kappa}=\mathbb{M}$ and $\mathbb{M}_{\kappa} \bigcap \mathbb{M}_{\kappa^{\prime}}=\{\}$ for $\kappa \neq \kappa^{\prime}$, and define a $\mathbb{K}$-valued aggregation mode process $\left\{\kappa_{t}\right\}$ as follows:

$$
\kappa_{t}(\omega)=\kappa, \text { if } \theta_{t}(\omega) \in \mathbb{M}_{\kappa}
$$

Because the evolution of the aggregation mode process $\left\{\kappa_{t}\right\}$ depends of the evolution of $\left\{\theta_{t}\right\},\left\{\kappa_{t}\right\}$ may inherit rare mode switching from $\left\{\theta_{t}\right\}$. In order to avoid these rare effects in the evolution of particles, we also define a $\mathbb{K}$-valued Markov chain $\left\{\breve{\kappa}_{t}\right\}$ with known non-rare transition rates, and use the transition rates of $\left\{\breve{\kappa}_{t}\right\}$ to determine for each particle a new $\breve{\kappa}$-value at some time step $h$ later. The particle weight is compensated with the corresponding importance switching ratio $p_{\kappa_{\tau+h} \mid \kappa_{\tau}, x_{\tau}, \theta_{\tau}}(\breve{\kappa} \mid \kappa, x, \theta) / p_{\breve{\kappa}_{\tau+h} \mid \breve{K}_{\tau}}(\breve{\kappa} \mid \kappa)$, where $\kappa, x, \theta$ denote the given $\left(\kappa_{\tau}, x_{\tau}, \theta_{\tau}\right)$ particle value, and $\breve{\kappa}$ denotes the value newly sampled for $\breve{\boldsymbol{K}}_{\tau+h}$.

Next, the prediction of the new $\theta_{\tau+h}$ particle from the $\left(x_{\tau}, \theta_{\tau}\right)$ particle values is done conditional on the newly sampled $\breve{\kappa}$ value. Theorem 1 provides a probabilistic characterization of such $\breve{\kappa}$-conditional $\theta$-prediction.

Theorem 1 ( $\breve{\kappa}$-conditional $\theta$-prediction)

Let $\tau$ be an arbitrary stopping time, then

$$
p_{\theta_{\tau+h} \mid x_{\tau}, \theta_{\tau}, \kappa_{\tau+h}}(\eta \mid x, \theta, \breve{\kappa})=\frac{1_{\mathbb{M}_{\tilde{\kappa}}}(\eta) p_{\theta_{\tau+h} \mid x_{\tau}, \theta_{\tau}}(\eta \mid x, \theta)}{\sum_{\eta^{\prime} \in \mathbb{M}} 1_{\mathbb{M}_{\tilde{\kappa}}}\left(\eta^{\prime}\right) p_{\theta_{\tau+h} \mid x_{\tau}, \theta_{\tau}}\left(\eta^{\prime} \mid x, \theta\right)}
$$

Proof: Using Bayes yields:

$$
\begin{aligned}
p_{\theta_{\tau+h} \mid x_{\tau}, \theta_{\tau}, \kappa_{\tau+h}}( & \eta \mid x, \theta, \breve{\kappa})= \\
& \frac{p_{\kappa_{\tau+h} \mid \theta_{\tau+h}}(\breve{\kappa} \mid \eta) p_{\theta_{\tau+h} \mid x_{\tau}, \theta_{\tau}}(\eta \mid x, \theta)}{\sum_{\eta^{\prime} \in \mathbb{M}} p_{\kappa_{\tau+h} \mid \theta_{\tau+h}}\left(\breve{\kappa} \mid \eta^{\prime}\right) p_{\theta_{\tau+h} \mid x_{\tau}, \theta_{\tau}}\left(\eta^{\prime} \mid x, \theta\right)}
\end{aligned}
$$

Substituting $p_{\kappa_{\tau+h} \mid \theta_{\tau+h}}(\breve{K} \mid \eta)=1_{\mathbb{M}_{\bar{\kappa}}}(\eta)$ yields (13).

Q.E.D.

The prediction of the $x$-part of the particle over time step $h$ is done by drawing a sample from $p_{x_{\tau+h} \mid x_{\tau}, \theta_{\tau}, \theta_{\tau+h}}(. \mid x, \theta, \eta)$.
In order to identify all particles that arrive at $Q_{k}$ before time $T$, the prediction over time step $h$ has to be done up to $T / h$ times. After these prediction steps, there is no guarantee that for each $\breve{\kappa} \in \mathbb{K}$ some minimum number of particles have arrived at $Q_{k}$. Hence we resample the $Q_{k}$-arrived particles such that we regain $N_{p}$ particles for each $\kappa \in \mathbb{K}$. In order to make this possible, in Theorem 2 we provide a characterization of the (conditional) probabilities $p_{\kappa_{\tau+h}}$ and $p_{x_{\tau}, \theta_{\tau} \mid \kappa_{\tau+h}}$ as a function of $p_{x_{\tau}, \theta_{\tau}}$, for arbitrary stopping time $\tau$ and time step $h$. This characterization allows to sample a fixed number of particles per aggregation mode $\kappa \in \mathbb{K}$, and to sample for each particle a novel $\theta$ value conditional on the aggregation mode value.

Theorem 2 (Hierarchical interaction)

If $p_{\kappa_{\tau+h}}(\kappa)>0$ for arbitrary stopping time $\tau$, then

$$
\begin{gathered}
p_{x_{\tau}, \theta_{\tau} \mid \kappa_{\tau+h}}(d x, \theta \mid \kappa)=\sum_{\eta \in \mathbb{M}_{\kappa}} p_{\theta_{\tau+h} \mid x_{\tau}, \theta_{\tau}}(\eta \mid x, \theta) . \\
\cdot p_{x_{\tau}, \theta_{\tau}}(d x, \theta) / p_{\kappa_{\tau+h}}(\kappa) \\
p_{\kappa_{\tau+h}}(\kappa)=\sum_{\theta \in \mathbb{M}} \int_{\mathbb{R}^{n}} \sum_{\eta \in \mathbb{M}_{\kappa}} p_{\theta_{\tau+h} \mid x_{\tau}, \theta_{\tau}}(\eta \mid x, \theta) . \\
. p_{x_{\tau}, \theta_{\tau}}(d x, \theta)
\end{gathered}
$$

Proof:

By definition of the partitioning $\left\{\mathbb{M}_{\kappa}, \kappa \in \mathbb{K}\right\}$ we have

$$
\begin{array}{r}
p_{\kappa_{\tau+h}, x_{\tau}, \theta_{\tau}}(\kappa, d x, \theta)=\sum_{\eta \in \mathbb{M}_{\kappa}} p_{\theta_{\tau+h}, x_{\tau}, \theta_{\tau}}(\eta, d x, \theta) \\
=\sum_{\eta \in \mathbb{M}_{\kappa}} p_{\theta_{\tau+h} \mid x_{\tau}, \theta_{\tau}}(\eta \mid x, \theta) p_{x_{\tau}, \theta_{\tau}}(d x, \theta)
\end{array}
$$

Dividing left and right hand sides by $p_{\kappa_{\tau+h}}(\kappa)$ yields (8).

From the law of total probability we have:

$$
p_{\kappa_{\tau+h}}(\kappa)=\sum_{\theta \in \mathbb{M}} \int_{\mathbb{R}^{n}} p_{\kappa_{\tau+h}, x_{\tau}, \theta_{\tau}}(\eta, d x, \theta)
$$

Substitution of the latter in the former yields (9). Q.E.D.

In order to see what Theorem 2 means for the empirical kind of densities that will be used, we assume $p_{x_{\tau}, \theta_{\tau}}($. equals an empirical density:

$$
p_{x_{\tau}, \theta_{\tau}}(d x, \theta)=\sum_{\kappa \in \mathbb{K}} \sum_{i=1}^{N^{K}} \omega^{\kappa, i} \delta_{\left(x^{K, i}, \theta^{\kappa, i}\right)}(d x, \theta)
$$

with $\left\{x^{\kappa, i}, \theta^{\kappa, i}, \omega^{\kappa, i}\right\}_{i=1}^{N^{\kappa}}, \kappa \in \mathbb{K}$, a given set of particles. Substituting (10) into (8) and evaluation yields: 


$$
\begin{gathered}
p_{x_{\tau}, \theta_{\tau} \mid \kappa_{\tau+h}}(d x, \theta \mid \kappa)=\sum_{\eta \in \mathbb{M}_{\kappa}} p_{\theta_{\tau+h} \mid x_{\tau}, \theta_{\tau}}(\eta \mid x, \theta) . \\
\cdot \sum_{\kappa^{\prime} \in \mathbb{K}} \sum_{i=1}^{N^{\kappa^{\prime}}} \omega^{\kappa^{\prime}, i} \delta_{\left(x^{\kappa^{\prime}, i}, \theta^{\kappa^{\prime}, i}\right)}(d x, \theta) / p_{\kappa_{\tau+h}}(\kappa) \\
=\sum_{\kappa^{\prime} \in \mathbb{K}} \sum_{i=1}^{N^{\kappa^{\prime}}} \sum_{\eta \in \mathbb{M}_{\kappa}} p_{\theta_{\tau+h} \mid x_{\tau}, \theta_{\tau}}\left(\eta \mid x^{\kappa^{\prime}, i}, \theta^{\kappa^{\prime}, i}\right) . \\
\cdot \omega^{\kappa^{\prime}, i} \delta_{\left(x^{\kappa^{\prime}, i}, \theta^{\kappa^{\prime}, i}\right)}(d x, \theta) / p_{\kappa_{\tau+h}}(\kappa)
\end{gathered}
$$

Similarly, substituting (10) into (9) yields

$$
p_{\kappa_{\tau+h}}(\kappa)=\sum_{\kappa^{\prime} \in \mathbb{K}} \sum_{i=1}^{N^{\kappa^{\prime}}} \sum_{\eta \in \mathbb{M}_{\kappa}} p_{\theta_{\tau+h} \mid x_{\tau}, \theta_{\tau}}\left(\eta \mid x^{\kappa^{\prime}, i}, \theta^{\kappa^{\prime}, i}\right) \omega^{\kappa^{\prime}, i}
$$

The idea is to use equation (11) for resampling $N_{p}$ particles from $p_{\tau_{\tau_{k}}, \theta_{\tau_{k}} \mid \kappa_{\tau_{k}+h}}(. \mid \kappa)$ for each $\kappa$-value once at the beginning of a prediction cycle from $\tau_{k}$ to $\tau_{k+1}$. Equation (12) is used to compensate each particle weight for this resampling.

\section{HIERARCHICAL Hybrid IPS AlgORITHM}

Similar as in the IPS algorithm for an SHS [12-13], a particle is defined as a triplet $(x, \theta, \omega), \omega \in[0,1]$, $x \in \mathbb{R}^{n}$ and $\theta \in \mathbb{M}$. Numerical approximations $\tilde{\gamma}_{k}$ and $\tilde{\pi}_{k}$ are used for $\gamma_{k}$ and $p_{\xi_{k} \mid \chi_{k}}(. \mid 1)$ respectively. When simulating from $\tau_{k-1} \wedge T$ to $\tau_{k} \wedge T$, a fraction $\tilde{\gamma}_{k}$ of the Monte Carlo simulated trajectories only will reach $Q_{k}$. The Hierarchical Hybrid Interacting Particle System (HHIPS) algorithm estimates these fractions and their product in a recursive way, using the following steps:

$\underline{\text { Step } 0}$ generates per $\kappa$-value $N_{p}$ initial particles at $k=0$, and then starts the cycling through steps 1 through 3 for $k:=1,2, \ldots m$.

Step 1 extrapolates each particle from $\tau_{k-1} \wedge T$ to $\tau_{k} \wedge T$ in time steps of length $h$, using importance switching for the new $\kappa$-value and $\kappa$-conditional sampling of a new $\theta$ value. For the latter use is made of the $\kappa$-conditional $\theta$-prediction characterization in Theorem 1.

$\underline{\text { Step } 2}$ evaluates the particles that have arrived at $Q_{k}$.

For this, use is made of equations (4)-(5).

Step 3 resamples from the particles that have arrived at $Q_{k}$. In order to draw $N_{p}$ samples per $\kappa$-value, use is made of the hierarchical interaction characterization in Theorem 2.

Each of these steps is specified in detail below.

\section{Hierarchical Hybrid Interacting Particle System \\ (HHIPS) Step 0: Initial sampling for $k=0$.}

- At time $t=0$ we start with a set of $N^{K}:=N_{p}$ particles for each aggregation mode $\kappa \in \mathbb{K}$ :

$\left\{x^{\kappa, i}, \theta^{\kappa, i}, \omega^{\kappa, i}\right\}_{i=1}^{N_{p}}, \kappa \in \mathbb{K}$, where the particles are obtained as follows.

First $\theta^{\kappa, i}$ are independently drawn from $p_{\theta_{0} \mid \kappa_{0}}(\cdot \mid \kappa)$.

Then $x^{\kappa, i} \in\{0\} \times \mathbb{R}^{n-1} / D_{1}$ are independently drawn from $p_{x_{0} \mid \theta_{0}}\left(\cdot \mid \theta^{\kappa, i}\right)$ with the first component of $x^{\kappa, i}$ equal to zero. The initial weights satisfy

$$
\omega^{\kappa, i}=\frac{p_{\kappa_{0}}(\kappa)}{N_{p}}, i=1, \ldots, N_{p}, \kappa \in \mathbb{K} .
$$

- With this we have $\tilde{\gamma}_{0}=1$ and

$$
\tilde{p}_{x_{0}, \theta_{0}}(d x, \theta)=\sum_{\kappa \in \mathbb{K}} \sum_{i=1}^{N^{\kappa}} \omega^{\kappa, i} \delta_{\left(x^{\kappa, i}, \theta^{\kappa, i}\right)}(d x, \theta)
$$

- Choose a sufficiently large number $J$ of equal discretization steps of time length $h=T / J$, which allows to use a numerical integration time step $h$.

- Choose an appropriate positive value for $\alpha<1 / J$.

HHIPS iteration cycle: For $k=1, \ldots, m$ cycle over step 1 (prediction), step 2 (assessment) and step 3 (resampling):

\section{HHIPS Step 1. Prediction:}

- Start with empty sets $S_{k}^{\kappa}, \kappa \in \mathbb{K}$, to store all particles that arrive at $Q_{k}=(0, T) \times D_{k} \times \mathbb{M}$.

- For $j=1, \ldots, J$, iterate over substeps 1.a, 1.b and 1.c.

Substep 1.a Sample $\kappa_{\tau+h}$ using importance switching. If $k>1$ and $j=1$, then goto substep $1 \mathrm{~b}$, else for each $\kappa \in \mathbb{K}$ and $i=1, \ldots, N^{\kappa}$ :

- If $\omega^{\kappa, i}=0$ then $\breve{\omega}^{\kappa, i}:=\omega^{\kappa, i}$ and $\breve{\kappa}^{\kappa, i}:=\kappa$; else, sample a $\breve{\kappa}^{\kappa, i} \in \mathbb{K}$ with probability $\alpha$ for each of the values in $\mathbb{K} /\{K\}$, and with probability $1-\alpha(|\mathbb{K}|-1)$ for the value $K$, and correct the corresponding weight according to this importance switching, i.e. 


$$
\breve{\omega}^{\kappa, i}= \begin{cases}\omega^{\kappa, i} \frac{p_{\kappa_{\tau+h} \mid x_{\tau}, \theta_{\tau}}\left(\breve{\kappa}^{\kappa, i} \mid x^{\kappa, i}, \theta^{\kappa, i}\right)}{1-\alpha(|\mathbb{K}|-1)} & \text { if } \breve{\kappa}^{\kappa, i}=\kappa \\ \omega^{\kappa, i} \frac{p_{\kappa_{\tau+h} \mid x_{\tau}, \theta_{\tau}}\left(\breve{\kappa}^{\kappa, i} \mid x^{\kappa, i}, \theta^{\kappa, i}\right)}{\alpha} & \text { if } \breve{\kappa}^{\kappa, i} \neq \kappa\end{cases}
$$

- The resulting sets of particles are $\left\{\bar{x}^{\kappa^{\prime}, l}, \bar{\theta}^{\kappa^{\prime}, l}, \breve{\omega}^{\kappa^{\prime}, l}, \breve{\kappa}^{\kappa^{\prime}, l}\right\}_{l=1}^{N^{\kappa^{\prime}}}, \kappa^{\prime} \in \mathbb{K} . \quad$ For $\quad$ each $\kappa \in \mathbb{K}$, collect from these particles those $N^{\kappa}$ particles for which $\breve{\kappa}^{\kappa^{\prime}, l}=\kappa$, i.e. $N^{\kappa}:=\sum_{\substack{\kappa^{\prime} \in \mathbb{K} \\ N^{\kappa^{\prime}} \neq 0}} \sum_{l=1}^{N^{\kappa^{\prime}}} 1_{\{\kappa\}}\left(\breve{\kappa}^{\kappa^{\prime}, l}\right)$.

- For each $\kappa \in \mathbb{K}$, renumber the indices of these $N^{K}$ particles such that the first index equals $K$ and the second index runs over $\left\{1, \ldots, N^{\kappa}\right\}$. This yields for each $\kappa \in \mathbb{K}$ the following new set of particles $\left\{x^{\kappa, i}, \theta^{\kappa, i}, \omega^{\kappa, i}\right\}_{i=1}^{N^{\kappa}}$ if $N^{\kappa} \neq 0$, and an empty set \{\} if $\mathrm{N}^{\kappa}=0$.

Substep 1.b $\kappa_{\tau+h}$-conditional prediction of $\left(x_{\tau+h}, \theta_{\tau+h}\right)$. For each $\kappa \in \mathbb{K}$, determine the new set of particles $\left\{\bar{x}^{\kappa, i}, \bar{\theta}^{\kappa, i}, \bar{\omega}^{\kappa, i}\right\}_{i=1}^{N^{\kappa}}$ as follows:

- For each $\kappa, i$ for which $\omega^{\kappa, i}=0$, set $\bar{x}^{\kappa, i}:=x^{\kappa, i}$ and $\bar{\theta}^{\kappa, i}:=\theta^{\kappa, i}$. Else, use Theorem 1 to sample a new value $\bar{\theta}^{\kappa, i}$ from:

$$
\begin{aligned}
p_{\theta_{\tau+h} \mid x_{\tau}, \theta_{\tau}, \kappa_{\tau+h}} & \left(\eta \mid x^{\kappa, i}, \theta^{\kappa, i}, \kappa\right)= \\
= & \frac{1_{\mathbb{M}_{\kappa}}(\eta) p_{\theta_{\tau+h} \mid x_{\tau}, \theta_{\tau}}\left(\eta \mid x^{\kappa, i}, \theta^{\kappa, i}\right)}{\sum_{\eta^{\prime} \in \mathbb{M}} 1_{\mathbb{M}_{\kappa}}\left(\eta^{\prime}\right) p_{\theta_{\tau+h} \mid x_{\tau}, \theta_{\tau}}\left(\eta^{\prime} \mid x^{K, i}, \theta^{K, i}\right)}
\end{aligned}
$$

and a new value $\bar{x}^{\kappa, i}$ from

$$
p_{x_{\tau+h} \mid \theta_{\tau+h}, x_{\tau}, \theta_{\tau}}\left(d x \mid \bar{\theta}^{\kappa, i}, x^{\kappa, i}, \theta^{\kappa, i}\right) \text {. }
$$

- The weights are not changed, i.e. $\bar{\omega}^{\kappa, i}:=\omega^{\kappa, i}$.

Substep 1.c. Memorizing particles that arrived at $Q_{k}$ :

- If $\left(\bar{x}^{\kappa, i}, \bar{\theta}^{\kappa, i}\right) \in Q_{k}$ and $\omega^{\kappa, i} \neq 0$, then a copy of the particle $\left\{\bar{x}^{\kappa, i}, \bar{\theta}^{\kappa, i}, \bar{\omega}^{\kappa, i}\right\}$ is stored in the set $S_{k}^{\kappa}$.

- Subsequently, we set $\bar{\omega}^{\kappa, i}:=0$ in the original particle.

- If $j=J$, then step 1 is complete, hence go to step 2, else, repeat substeps $1 \mathrm{a}, \mathrm{b}, \mathrm{c}$ for $j:=j+1$.
HHIPS Step 2. Evaluate the $Q_{k}$ arrived particles:

- The particles which are memorized in $S_{k}^{\kappa}, \kappa \in \mathbb{K}$, provide an estimate of $p_{\xi_{k} \mid \chi_{k}}(. \mid 1)$ and $\gamma_{k}$.

- Renumbering the particles in $S_{k}^{K}$ yields a set of particles $\left\{\tilde{x}^{\kappa, i}, \tilde{\theta}^{\kappa, i}, \tilde{\omega}^{\kappa, i}\right\}_{i=1}^{N^{\kappa}}$ with $N^{\kappa}$ the number of particles in $S_{k}^{\kappa}$.

- Weighted fraction $\tilde{\gamma}_{k}$ of the $Q_{k}$ arrived particles:

$$
\gamma_{k} \approx \tilde{\gamma}_{k}=\sum_{\substack{\kappa \in \mathbb{K} \\ N^{\kappa} \neq 0}} \sum_{i=1}^{N^{\kappa}} \tilde{\omega}^{\kappa, i}
$$

- If $N^{\kappa}=0$ for all $\kappa \in \mathbb{K}$, then the algorithm stops with estimate $P_{h i t}(0, T) \approx 0$.

- If $k=m$, then stop HHIPS with the estimate $P_{h i t}(0, T) \approx \prod_{k=1}^{m} \tilde{\gamma}_{k}$.

- For each $\kappa \in \mathbb{K}$ and $i=1, \ldots, N^{\kappa}$ : $\tilde{\omega}^{\kappa, i}:=\tilde{\omega}^{\kappa, i} / \tilde{\gamma}_{k}$

- The estimated $p_{\xi_{k} \mid \chi_{k}}(. \mid 1)$ satisfies:

$p_{\xi_{k} \mid \chi_{k}}(d x, \theta \mid 1) \approx \tilde{\pi}_{k}(d x, \theta)=\sum_{\substack{\kappa \in \mathbb{K} \\ N^{k} \neq 0}} \sum_{i=1}^{N^{\kappa}} \tilde{\omega}^{\kappa, i} \delta_{\left(\tilde{x}^{\kappa, i}, \tilde{\theta}^{\kappa, i}\right)}(d x, \theta)$

HHIPS Step 3. Copy the $Q_{k}$ arrived particles through $\boldsymbol{\kappa}_{\tau_{k}+h}$-conditional resampling.

- Evaluate aggregated mode probabilities at $\tau:=\tau_{k}$ using (12):

$$
\begin{aligned}
& p_{\kappa_{\tau+h} \mid \chi_{k}}(\kappa \mid 1) \approx \varphi(\kappa)= \\
& =\sum_{\substack{\kappa^{\prime} \in \mathbb{K} \\
N^{\kappa^{\prime} \neq 0}}} \sum_{i=1}^{N^{\kappa^{\prime}}} \sum_{\eta \in \mathbb{M}_{\kappa^{\prime}}} p_{\theta_{\tau+h} \mid x_{\tau}, \theta_{\tau}}\left(\eta \mid \tilde{x}^{\kappa^{\prime}, i}, \tilde{\theta}^{\kappa^{\prime}, i}\right) \tilde{\omega}^{\kappa^{\prime}, i}
\end{aligned}
$$

- For each $\kappa \in \mathbb{K}$ independently draw $N_{p}$ random pairs $\left(x^{\kappa, i}, \theta^{\kappa, i}\right), \quad i=1, \ldots, N_{p}$ from the particle spanned empirical measure, using (11): 


$$
\begin{aligned}
& p_{x_{\tau}, \theta_{\tau} \mid \kappa_{\tau+h}, \chi_{k}}(d x, \theta \mid \kappa, 1) \approx \\
& \approx \sum_{\kappa^{\prime} \in \mathbb{K}} \sum_{i=1}^{N^{\kappa^{\prime}}} \sum_{\eta \in \mathbb{M}_{\kappa^{\prime}}} p_{\theta_{\tau+h} \mid x_{\tau}, \theta_{\tau}}\left(\eta \mid \tilde{x}^{\kappa^{\prime}, i}, \tilde{\theta}^{\kappa^{\prime}, i}\right) . \\
& . \tilde{\omega}^{\kappa^{\prime}, i} \delta_{\left\{\tilde{x}^{\kappa^{\prime}, i}, \tilde{\theta}^{\kappa^{\prime}, i}\right\}}(d x, \theta) / \varphi(\kappa)
\end{aligned}
$$

- This yields, for each $\kappa \in \mathbb{K}$, a set of particles $\left\{x^{\kappa, i}, \theta^{\kappa, i}, \omega^{\kappa, i}\right\}_{i=1}^{N_{p}}$ with $\omega^{\kappa, i}:=\varphi(\kappa) / N_{p}$.

- If $k<m$, then repeat steps $1-3$ for $k:=k+1$ and $N^{\kappa}:=N_{p}$

Remark: The key extensions of HHIPS over IPS for an SHS [12-13] are:

1. Embedding of an aggregation mode process;

2. Particles are maintained per aggregation mode;

3. Importance switching of aggregation mode is used for the conditional prediction of SHS particles;

4. Hierarchical interaction is used for the resampling of particles that reached $Q_{k}, k=1, . ., m-1$.

\section{FreE Flight AIR TRAFFIC EXAMPLE}

We consider a specific free flight operational concept that has been developed within a recent European research project [22]. In order to use MC simulation for the estimation of safety risk, we first developed a MC simulator of these operations such that the simulated trajectories constitute executions of a generalized SHS [23]. The dimensionality of the resulting MC simulation model is very large, e.g. in simulating two aircraft there are about $10^{25}$ discrete mode combinations, and the Euclidean state may go up to $\mathbb{R}^{336}$ [12]. In [12]-[13], [24] we developed a way to cast the air traffic SHS model within the setting of the IPS formulation, and used the IPS to evaluate demanding high risk bearing multi-aircraft scenarios. This IPS approach, however, did not work properly anymore for low risk bearing scenarios. The aim of this section is to demonstrate that the novel HHIPS works well for such a low risk bearing scenario, using the same SHS model.

The $D_{k}$ 's are defined by three parameters, the values of which are given in Table 1 for a sequence of eight nested subsets. Here $d_{k}$ and $h_{k}$ define a cylinder of diameter $d_{k}$ and height $h_{k}$ respectively. The $\Delta_{k}$ value is the time period over which position and velocity differences between the two aircraft are compared. If within $\Delta_{k}$ the predicted position difference falls within the corresponding cylinder, then $D_{k}$ is said to be reached. The three parameters of $D_{8}$ are such that its reaching represents a collision between the aircraft.
TABLE 1

IPS CONFLICT LEVEL PARAMETER VALUES

\begin{tabular}{|l|c|c|c|c|c|c|c|c|}
\hline$k$ & 1 & 2 & 3 & 4 & 5 & 6 & 7 & 8 \\
\hline $\begin{array}{l}d_{k} \\
\mathrm{Nm}\end{array}$ & 5.0 & 5.0 & 5.0 & 5.0 & 2.5 & 1.25 & .50 & .054 \\
\hline $\begin{array}{l}h_{k} \\
\mathrm{ft}\end{array}$ & 1000 & 1000 & 1000 & 1000 & 1000 & 500 & 250 & 131 \\
\hline $\begin{array}{c}\Delta_{k} \\
\min \end{array}$ & 8 & 2.5 & 1.5 & 0 & 0 & 0 & 0 & 0 \\
\hline
\end{tabular}

In the low risk bearing scenario considered, two aircraft start at the same flight level, some $250 \mathrm{~km}$ away from each other, and fly on opposite direction flight plans head-on with a ground speed of $240 \mathrm{~m} / \mathrm{s}$. This means that collision may be reached after about 500s simulation, hence we set $T$ $=600 \mathrm{~s}$. The collision reach probability is estimated through running ten times the HHIPS algorithm ${ }^{1}$. The aggregation modes chosen are all combinations of the following high level mode values: global communication support is 'up' or 'down', and decision-making (DM) loop of aircraft 1 is 'up' or 'down'. This leads to a total of four aggregation mode values. The number of particles used is 5,000 per aggregation mode value; hence 20,000 particles are used per HHIPS run. The time step $h=1$ s, and $\alpha=0.001$. Results of these HHIPS runs are presented in Tables II-IV.

Table II: $\tilde{\gamma}_{k}$ values estimated by first four HHIPS runs. IPS based estimation typically yields values 0.0 for $k \geq 4$.

\begin{tabular}{|c|c|c|c|c|}
\hline$k$ & Run 1 & Run 2 & Run 3 & Run 4 \\
\hline $\mathbf{1}$ & 0.993 & 0.992 & 0.999 & 0.999 \\
\hline $\mathbf{2}$ & 0.295 & 0.280 & 0.289 & 0.294 \\
\hline $\mathbf{3}$ & 0.040 & 0.048 & 0.050 & 0.047 \\
\hline $\mathbf{4}$ & $2.71 \mathrm{E}-4$ & $2.59 \mathrm{E}-4$ & $2.69 \mathrm{E}-4$ & $2.70 \mathrm{E}-4$ \\
\hline $\mathbf{5}$ & 0.206 & 0.143 & 0.118 & 0.233 \\
\hline $\mathbf{6}$ & 0.427 & 0.539 & 0.527 & 0.396 \\
\hline $\mathbf{7}$ & 0.855 & 0.858 & 0.889 & 0.759 \\
\hline $\mathbf{8}$ & 0.810 & 0.823 & 0.827 & 0.754 \\
\hline $\boldsymbol{\Pi}$ & $1.93 \mathrm{E}-7$ & $1.86 \mathrm{E}-7$ & $1.76 \mathrm{E}-7$ & $1.96 \mathrm{E}-7$ \\
\hline
\end{tabular}

Table II presents the values for $\tilde{\gamma}_{k}$ which have been estimated during the first four HHIPS runs. The estimated mean probability of collision between the two aircraft equals $1.9 \times 10^{-7}$. The estimated standard deviation is $0.8 \times 10^{-8}$, which shows that the estimated value is quite accurate. It should be noticed that the variation in the fractions per level is significantly larger than the variation in the product of the fractions. Apparently, the dependency between the fractions $\tilde{\gamma}_{k}$ reduces the variation in the

\footnotetext{
${ }^{1}$ In [25] a similar kind of two aircraft encounter scenario has been simulated using a heuristic precursor of the current HHIPS
} 
multiplication of these fractions.

Table III shows the percentage in contribution to collision reach probability for each of the four aggregation mode values. This shows that the risk contribution is almost completely caused by "global communication down".

Table III. Contribution to reach probability.

\begin{tabular}{|c|c|c|}
\hline $\begin{array}{c}\text { Global } \\
\text { comm. }\end{array}$ & DM-loop & $\begin{array}{c}\text { Share } \\
\mathbf{\%}\end{array}$ \\
\hline $\mathrm{Up}$ & $\mathrm{Up}$ & 0.5 \\
\hline $\mathrm{Up}$ & Down & 1.1 \\
\hline Down & $\mathrm{Up}$ & 98.4 \\
\hline Down & Down & 0.002 \\
\hline
\end{tabular}

Finally we improved the availability/reliability of the ASAS related systems by a factor 100 , and then conducted the ten HHIPS runs again. This resulted in a 100-fold decrease of the collision reach probability. These results demonstrate that HHIPS works well for this large scale SHS.

Ackowledgement: The first author of this paper likes to thank Pierre Del Moral (Univ. of Nice), Francois LeGland and Frederic Cerou (both of INRIA/IRISA, Rennes) and Pascal Lezaud (CENA/ENAC, Toulouse) for valuable discussions of their work.

\section{REFERENCES}

[1] S. Amin, A. Abate, M. Prandini, J. Lygeros, S. Sastry, Reachabiliy analysis for controlled discrete time stochastic hybrid systems, Eds: J. Hespanha and A. Tiwari, Proc. HSCC2006, Lecture Notes in Computer Science, Springer, 2006, pp. 49-63.

[2] A. Abate, S. Amin, M. Prandini, J. Lygeros, S. Sastry, Probabilistic reachabiliy for discrete time stochastic hybrid systems, Proc. IEEE Conference on Decision and Control, December 2006, pp. 258-263.

[3] M. Prandini, J. Hu. A stochastic approximation method for reachability computations. Eds: H.A.P. Blom, J. Lygeros, Stochastic Hybrid Systems, Theory and safety critical applications, Springer, Berlin, July 2006, pp. 107-139.

[4] J.A. Bucklew, Introduction to rare event estimation, Springer, 2004.

[5] T.E. Booth, Monte Carlo variance comparison for expected-value versus sampled splitting, Nuclear Science and Engineering, Vol. 89 (1985), pp. 305-309.

[6] O. Atkin, J.K. Townsend, Efficient simulation of TCP/IP networks characterzed by non-rare events using DPR-based splitting, Proc. IEEE Globecom, 2001, pp. 1734-1740.

[7] P. L'Ecuyer, V. Demers and B. Tuffin, Splitting for rare-event simulation, Proc. 2006 Winter Simulation Conference, Eds: L.F Perrone et al., IEEE December 2006, pp. 137-148.

[8] F. Cerou, P. Del Moral, F. LeGland and P. Lezaud, Genetic genealogical models in rare event analysis, Publications du Laboratoire de Statistiques et Probabilites, Toulouse III, 2002, available at http://www.nlr.nl/public/hosted-sites/hybridge/

[9] P. Del Moral, Feynman-Kac Formulae. Genealogical and Interacting Particle Systems with Applications, Springer, 2004.

[10] F. Cerou, P. Del Moral, F. LeGland, P. Lezaud, Limit theorems for the multi-level splitting algorithms in the simulation of rare events, Proc. 2005 Winter Simulation Conference, Orlando, USA.

[11] P. Del Moral, P. Lezaud. Branching and interacting particle interpretations of rare event probabilities. Eds: H.A.P. Blom, J.
Lygeros, Stochastic Hybrid Systems, Theory and safety critical applications, Springer, Berlin, 2006, pp. 277-324.

[12] H.A.P. Blom, J. Krystul, G.J. Bakker, M.B. Klompstra, B. KleinObbink, Free flight collision risk estimation by sequential Monte Carlo simulation, Eds: C.G. Cassandras, J. Lygeros, Stochastic Hybrid Systems, CRC Press/Taylor\&Francis, November 2006, pp. 249-281.

[13] H.A.P. Blom, J. Krystul, G.J. Bakker, Safety verification of free flight in air traffic, Proc. IEEE Conf. on Decision and Control, San Diego, CA, December 13-15, 2006.

[14] J. Krystul and H.A.P. Blom, Sequential Monte Carlo simulation of rare event probability in stochastic hybrid systems, Proc. $16^{\text {th }}$ IFAC World Congress, June 4-8, 2005, Prague, Czech Republic.

[15] J. Krystul, H.A.P. Blom. Sequential Monte Carlo simulation for the estimation of small reachability probabilities for stochastic hybrid systems. In Proceedings of IEEE-EURASIP Int. Symposium on Control, Communications and Signal Processing, Marrakech, Morocco, March 13-15, 2006.

[16] J. Krystul, PhD Thesis, Twente University, Enschede, The Netherlands, September 2006.

[17] M.J.J. Garvels, The splitting method in rare event simulation, $\mathrm{PhD}$ Thesis, Univ. Twente, 2000.

[18] M.J.J. Garvels, D.P. Kroese, J.K.C.W. van Ommeren, On the importance function splitting simulation, European Transactions on Telecommunications, Vol. 13 (2002), pp. 363-371.

[19] M.L. Bujorianu, Extended stochastic hybrid systems, Proc. Hybrid Systems Computation and Control 2004, Eds: O. Mahler, A Pnuelli, LNCIS number 2993, Springer, Berlin, 2003, pp. 234-249.

[20] M.L. Bujorianu, J. Lygeros. Towards a general theory of stochastic hybrid systems. Eds: H.A.P. Blom, J. Lygeros, Stochastic Hybrid Systems, Theory and safety critical applications, Springer, Berlin, 2006, pp. 3-30.

[21] J. Krystul, H.A.P. Blom, A. Bagchi, Stochastic differential equations on hybrid state space, Eds: C.G. Cassandras, J. Lygeros, Stochastic Hybrid Systems, Taylor and Francis, November 2006, chapter 2.

[22] F. Maracich, Flying free flight: pilot perspective and system integration requirement, IEEE Aerospace and Electronic Systems Magazine, July 2006, pp. 3-7.

[23] M.H.C. Everdij, M.B. Klompstra, H.A.P. Blom, B. Klein Obbink. Compositional specification of a multi-agent system by stochastically and dynamically coloured Petri nets. . Eds: H.A.P. Blom, J. Lygeros, Stochastic Hybrid Systems, Theory and Safety Critical Application, Springer, Berlin, 2006, pp. 325-350.

[24] H.A.P. Blom, G.J. Bakker, B. KleinObbink, M.B. Klompstra, Free flight safety risk modeling and simulation, Proc. $2^{\text {nd }}$ International Conference on Research in Air Transportation (ICRAT2006), Belgrade, Serbia, 26-28 ${ }^{\text {th }}$ June 2006.

[25] H.A.P. Blom, J. Krystul, G.J. Bakker, Estimating rare event probabilities in large scale stochastic hybrid systems by sequential Monte Carlo simulation, Proc. Int. Workshop on Rare Event SIMulation (RESIM), October 9-10, 2006, Bamberg, Germany.

[26] M.H.C. Everdij, H.A.P. Blom, Hybrid Petri nets that have intomappings with generalised stochastic hybrid processes, Eds: H.A.P. Blom, J. Lygeros, Stochastic Hybrid Systems, Theory and Safety Critical Application, Springer, Berlin, 2006, pp. 31-63.

[27] P.E. Labeau, C. Smidts and S. Swaminathan (2000). Dynamic reliability: towards an integrated platform for probabilistic risk assessment. Reliability Engineering and System Safety 68, 219-254.

[28] M.H.C. Everdij, H.A.P. Blom, G.J. (Bert) Bakker. Modeling lateral spacing and separation for airborne separation assurance using Petri nets. Simulation; Transactions of the Society for Modeling and Simulation International, accepted for publication in March 2006.

[29] P. Glasserman, P. Heidelberger, P. Shahabuddin, T. Zajic, Multilevel splitting for estimating rare event probabilities, Operations Research, Vol. 47 (1999), pp. 585-600.

[30] F. Cerou, P. Del Moral, F. LeGland, P. Lezaud, Genetic genealogical models in rare event analysis, Latin American Journal of Probability and Mathematical Statistics, ALEA, Vol. 1 (2006), pp. 181-203. 